\title{
Transmission spectral properties of clouds for hot Jupiter exoplanets
}

\begin{abstract}
H. R. Wakeford and D. K. Sing
Astrophysics Group, University of Exeter, Stocker Rd, Exeter, EX4 4QL, UK

e-mail: hannah@astro.ex.ac.uk

Received 14 May 2014 / Accepted 25 September 2014

ABSTRACT

Clouds play an important role in the atmospheres of planetary bodies. It is expected that, like all the planetary bodies in our solar system, exoplanet atmospheres will also have substantial cloud coverage, and evidence is mounting for clouds in a number of hot Jupiters. To better characterise planetary atmospheres, we need to consider the effects these clouds will have on the observed broadband transmission spectra. Here we examine the expected cloud condensate species for hot Jupiter exoplanets and the effects of various grain sizes and distributions on the resulting transmission spectra from the optical to infrared, which can be used as a broad framework when interpreting exoplanet spectra. We note that significant infrared absorption features appear in the computed transmission spectrum, the result of vibrational modes between the key species in each condensate, which can potentially be very constraining. While it may be hard to differentiate between individual condensates in the broad transmission spectra, it may be possible to discern different vibrational bonds, which can distinguish between cloud formation scenarios, such as condensate clouds or photochemically generated species. Vibrational mode features are shown to be prominent when the clouds are composed of small sub-micron sized particles and can be associated with an accompanying optical scattering slope. These infrared features have potential implications for future exoplanetary atmosphere studies conducted with JWST, where such vibrational modes distinguishing condensate species can be probed at longer wavelengths.
\end{abstract}

Key words. techniques: spectroscopic - planets and satellites: atmospheres

\section{Introduction}

Where there is an atmosphere, there are clouds, or so the evidence suggests. Every planet in our solar system with a persistent atmosphere has clouds, though they are notoriously hard to define. Here we take the definition of liquid or solid aerosol particles suspended in a planet's atmosphere. Clouds are a vital part of the energy balance of a planetary atmosphere, and they can potentially play a major role in the observational structure, blocking the atmosphere beneath them and weakening any emergent spectral lines. Jupiter's atmosphere is a prime example of how clouds can shape the atmosphere of a planet, forming coloured belts and zones of dark and light bands through vertical mixing of different species, which can represent differences in cloud depth of over one planetary scale height (Evans \& Hubbard 1972). The clouds in the atmospheres of solar system planets are, however, hugely different from what we expect to form in the atmospheres of hot Jupiters (e.g. Sudarsky et al. 2003; Marley et al. 2007; Lodders 2010).

Hot Jupiters occupy a vastly different region of parameter space compared to the planets in our solar system, occupying higher temperatures, spanning wider temperature regimes, pressure structures, and chemical compositions. To compute the expected transmission spectrum for different cloud condensates requires us to understand a number of different processes in a planetary atmosphere. Studies have been conducted on the impact of clouds in exoplanet atmospheres (e.g. Ackerman \& Marley 2001; Fortney 2005; Helling 2008; Howe \& Burrows 2012; Marley et al. 2013; Morley et al. 2013) and the condensates that are expected in a wide range of temperatures from brown dwarfs to solar system bodies (e.g. Burrows \& Sharp 1999; Khare et al. 2001; Lodders 2003; Cruikshank et al. 2005; Seager 2010; Morley et al. 2012).

Clouds and hazes in exoplanetary atmospheres can have a strong effect on the emerging spectra. As a principle source of irradiative scattering, their presence increases the reflected flux in the visible and near-infrared regions of the spectrum (Sudarsky et al. 2003). In addition, significant absorption features can be present (Morley et al. 2014). In principle, clouds and hazes can be the result of condensation chemistry or be photochemically produced. The solar system giant planets are likely dominated by photochemical stratospheric hydrocarbon hazes (Nixon et al. 2010) produced in a similar way to tholins in the atmosphere of Titan (Khare et al. 1984). The strong UV flux on the upper atmosphere of hot Jupiter exoplanets may generally enhance photochemically generated hydrocarbon species. However, studies by Liang et al. (2004) have found that the abundance of hydrocarbons in close-in giant planet atmospheres is significantly less than found in Jupiter and Saturn, where the high abundance of hydrocarbon aerosols results in strong absorption features shortwards of $600 \mathrm{~nm}$. The presence of non-equilibrium photochemical species with absorption in the blue gives their atmosphere a characteristic red colour (Zahnle et al. 2009). The planetary albedo, for instance the observed blue albedo measurement of HD 189733b (Evans et al. 2013), could help differentiate between strong Rayleigh scattering dust and red tholin-like species.

Recent studies of hot Jupiters have revealed that many of the exoplanets observed in transmission have cloudy or hazy properties, with their spectra dominated by strong optical Rayleigh 
and/or Mie scattering from high-altitude aerosol particles (Pont et al. 2008, 2013; Sing et al. 2009, 2011, 2013; Gibson et al. 2013). Clouds and hazes in the optical range effectively obscure any features from the deeper atmosphere, including pressurebroadened alkali $\mathrm{Na}$ and $\mathrm{K}$ lines and, in some cases, mute or completely cover expected water absorption features in the near infrared. Broadband transmission spectra of exoplanets, such as WASP-12b and HD 189733b, show strong scattering in the optical to near-infrared region of the spectrum (Redfield et al. 2008; Huitson et al. 2012). HD 189733b is one of the most extensively studied exoplanets to date, and in transmission the atmosphere is dominated by Rayleigh scattering over the whole visible range well into the infrared, with only narrow $\mathrm{Na}$ and $\mathrm{K}$ absorption line features detected above the Rayleigh slope (Pont et al. 2013).

Cloud composition and formation modelling for exoplanet and brown dwarf atmospheres show a number of different approaches to seed particle growth and transport. Models from Helling et al. (e.g. Helling 2007, 2009a,b) primarily use the topdown approach, which follows seed particle growth as it drops through the atmosphere accumulating condensates and fully accounts for the micro-physics of the grain growth. Ackerman \& Marley (2001) models consider the implications of downward transport of particles by sedimentation, balanced by upward mixing of vapour and condensates, in turn describing a mean global cloud in one-dimension. As a result, these two different approaches predict different cloud compositions for exoplanet atmospheres; only with more comparative studies and observations can differentiation between such models occur.

The location and formation of cloud condensates is informed by the temperature-pressure (T-P) profile of the exoplanetary atmosphere. The altitude, and therefore pressure, at which a cloud deck will be observed is dependent on the condensation temperature as a function of pressure, where the condensation curve crosses the planetary T-P profile (Marley et al. 2013; Morley et al. 2013). At and around the millibar pressure range it can be assumed, to the first order, that the temperature is constant with altitude at the limb of the planet when the absence of significant inversions is assumed (Fortney 2005).

Studies of dust species in the interstellar medium (ISM) have shown that while the precise composition cannot be determined from the absorption spectra alone (e.g. Li \& Draine 2001, 2002; Draine 2003), it is possible to differentiate between different bond species from their stretching mode frequency, which generates strong broad absorption features at characteristic wavelengths. Similar detections of broadband absorption features have been made in brown dwarf atmospheric spectra (Cushing et al. 2006; Burgasser et al. 2008), where absorption features observed in the mid infrared are attributed to clouds of small silicate grains in the photosphere of cloudy L dwarfs. A majority of the absorption and emission features of dust species in the ISM and brown dwarfs can be found between 3 and $25 \mu \mathrm{m}$. These features show strong correlation to wavelengths of major optically-active vibrational modes.

In this paper we discuss the radiative properties of multiple cloud condensates expected for hot Jupiter atmospheres and compute the expected transmission spectra over a wide wavelength regime, including well into the infrared. Condensate absorption properties rely upon the index of refraction which we discuss in Sect. 2.1 and later use to calculate the scattering and extinction cross-sections of each condensate. In Sect. 2.2 we discuss Mie Theory and bond species vibrational modes in the context of potentially observable condensates. These are then combined to calculate the transmission spectrum in Sect. 3 using the planetary scale height and condensate abundance with a look at the effect of different grainsize distributions. We apply the calculations to the well studied hot Jupiter HD 189733b to give reference to the resulting spectra. In Sect. 4 we discuss the results in context of future James Webb Space Telescope (JWST) observations and pay particular attention to species differentiation in small grainsize condensate transmission spectra.

\section{Radiative properties of cloud condensates}

Condensate chemistry in hot Jupiter atmospheres is dependent on the temperature-pressure profile and mass balance between refractory elements, such that the formation of condensate clouds at high temperatures severely depletes the gas at lower temperatures (Lodders 1999). The condensates considered in this paper are compiled from a number of sources considering equilibrium and condensate chemistry in brown dwarf and exoplanet atmospheres (e.g. Lodders 1999; Lodders \& Fegley Jr 2006; Helling 2008; Morley et al. 2012, 2013; see Table 1) with consideration for cooler atmospheres like those found in our solar system (e.g. Carlson et al. 1988; Baines et al. 1995; Lodders 2003; Bilger et al. 2013; see Table 2).

The condensates and cloud properties control the way that radiation moves through the planet's atmosphere, with the planetary transmission spectra dependent on the absorption and scattering of incoming and outgoing radiation. Mie theory is used to derive the absorption and scattering cross sections of solid and liquid particles (Hansen \& Travis 1974), which can then be used to estimate transmission spectra. In this study, we do not attempt to derive a full self-consistent cloud model, but rather use simple analytic formulae and the expectations of current cloud modelling to help interpret hot Jupiter spectra.

\subsection{Index of refraction}

In order to calculate all radiative properties of cloud condensates, knowledge of their refractive properties is needed. The experimental values of the refractive index need to be known so that accurate absorption cross sections can be calculated. Table 1 shows a list of cloud condensates and where the refractive indices for this work were obtained. The index of refraction is defined as $N=n+\mathrm{i} k$, where $n$ and $\mathrm{i} k$ are the real and complex parts of the refractive index, respectively. The index of refraction informs the scattering and absorption of electromagnetic waves through a material, while the complex index of refraction acts as a damping factor and is used to describe the attenuation of the waves (Liou 2002).

Figure 1 shows the real and complex index of refraction for each of the condensates in Table 1. The wavelength coverage of each condensate is determined by the experimental data presented in the associated papers. The calculated spectra are also dependent on the resolution of the measurements recorded. Features in the absorption properties of each condensate will have strong implications on the resulting spectrum of that particle. By referring to the complex index of refraction, trends emerge between different species of condensates, which can be explained by the vibrational properties of each molecule (see Sect. 3.2).

\subsection{Mie theory}

Mie theory is an analytical solution to Maxwell's equations, which describes how to calculate the phase functions and absorption and scattering cross sections of solid or liquid particles. 
H. R. Wakeford and D. K. Sing: Transmission spectral properties of clouds for hot Jupiter exoplanets

Table 1. References for $n$ and $k$ index for a number of condensates expected to form clouds in the upper atmosphere of hot Jupiters.

\begin{tabular}{ccccc}
\hline \hline Condensate & $\begin{array}{c}\text { Reference } \\
n, k \text { index }\end{array}$ & $\begin{array}{c}\lambda \text { range } \\
(\mu \mathrm{m})\end{array}$ & $\begin{array}{c}\text { Condensation } \\
\text { temperature } \\
(\mathrm{K})\end{array}$ & $\begin{array}{c}\text { Molecular } \\
\text { weight }\end{array}$ \\
\hline $\mathrm{SiO}_{2}$ & Palik (1998) & $0.04-11$ & 1725 & 60.08 \\
& Andersen et al. (2006) & $7-28$ & - & - \\
$\mathrm{Al}_{2} \mathrm{O}_{3}$ & M. Meinecke (2005)* & $6.6-10000$ & - & - \\
$\mathrm{FeO}$ & Koike et al. (1995) & $0.3-150$ & $1677^{1}$ & 101.96 \\
& Begemann et al. (1995) & $10-100$ & $1650^{4}$ & 71.79 \\
$\mathrm{CaTiO}_{3}$ & Andersen et al. (2006) & $15-40$ & - & - \\
$\mathrm{Fe}_{2} \mathrm{O}_{3}$ & Posch et al. (2003) & $2-155$ & $1582^{1}$ & 135.94 \\
$\mathrm{Fe}_{2} \mathrm{SiO}_{4}$ & M. Meinecke (2005)* & $0.1-987$ & 1566 & 159.68 \\
$\mathrm{MgAl}_{2} \mathrm{O}_{4}$ & Day (1981) & $8.2-35$ & $1443^{4}$ & 203.77 \\
$\mathrm{FeSiO}_{3}$ & M. Meinecke (2005)* & $1.6-270$ & $1397^{1}$ & 142.26 \\
$\mathrm{Mg}_{2} \mathrm{SiO}_{4}(\mathrm{Fe}-\mathrm{rich})$ & Day (1981) & $8.2-35$ & $1366^{4}$ & 131.92 \\
$\mathrm{Mg}_{2} \mathrm{SiO}_{4}(\mathrm{Fe}-$ poor) & Henning et al. (2005) & $0.2-445$ & $1354^{1}$ & 140.63 \\
$\mathrm{MgSiO}_{3}$ & Zeidler et al. (2011) & $0.19-800$ & $1354^{1}$ & 140.63 \\
& Egan \& Hilgeman (1975) & $0.1-0.4$ & $1316^{1}$ & 100.33 \\
$\mathrm{Na}_{2} \mathrm{~S}$ & Dorschner et al. (1995) & $0.5-80$ & - & - \\
$\mathrm{MnS}$ & Morley et al. (2012) & $0.03-73$ & 1176 & 78.04 \\
$\mathrm{TiO}_{2}$ & Huffman \& Wild (1967) & $0.1-3$ & $1139^{2}$ & 87.00 \\
& Kangarloo (2010a) & $0.3-1.2$ & $1125^{2}$ & 79.86 \\
$\mathrm{NaCl}_{\mathrm{KCl}}$ & Kangarloo (2010b) & $1.3-30$ & - & - \\
$\mathrm{ZnS}_{\mathrm{CH}}$ & Palik (1998) & $0.04-1000$ & $825^{3}$ & 58.44 \\
$\mathrm{C}_{6} \mathrm{H}_{12}$ & Palik (1998) & $0.02-200$ & $740^{3}$ & 74.55 \\
$\mathrm{Titan}_{\mathrm{Tholins}}$ & Querry (1987) & $0.2-167$ & $700^{5}$ & 97.45 \\
& Martonchik \& Orton (1994) & $0.02-72$ & $\sim 80$ & 16.04 \\
& Anderson (2000) & $2.0-25$ & 68 & 84.1 \\
& Khare et al. (1984) & $0.01-0.2$ & $\leq 90$ & $\sim 50.0$ \\
& Ramirez et al. (2002) & $1.1-1000$ & - & - \\
& $0.2-1$ & - & - \\
\hline
\end{tabular}

Notes. ${ }^{(*)}$ http: //www. astro. uni-jena.de/Laboratory/OCDB/oxsul.html; ${ }^{(+)}$at $10^{-3}$ bar.

References. ${ }^{(1)}$ Lodders (2003); ${ }^{(2)}$ Grossman (1972); ${ }^{(3)}$ Burrows \& Sharp (1999); ${ }^{(4)}$ Ebel \& Grossman (2000); (5) Morley et al. (2012).

To compute these we use bhmie ${ }^{1}$, an IDL routine that uses Bohren-Huffman Mie scattering to calculate scattering and absorption by a homogeneous isotropic sphere (Bohren \& Huffman 1983). When the radius of the particle greatly exceeds the wavelength, the theory tends to geometric optics, while if the wavelength greatly exceeds the radius of the particle Mie theory tends to Rayleigh scattering as observed in a number of exoplanetary atmospheres in the UV and optical (Lecavelier Des Etangs et al. 2008; Sing et al. 2011). Mie theory is used to derive solutions for spherical particles and is able to provide a first-order description of optical effects in non-spherical particles like those likely to exist in planetary atmospheres.

\subsubsection{Scattering and extinction efficiency}

Given the index of refraction, we can calculate the extinction, scattering, and absorption cross-section $\left(\sigma_{\mathrm{abs}}\right)$ and efficiency for a given particle size, where the scattering efficiency $\left(Q_{\text {scatt }}\right)$ and extinction efficiency $\left(Q_{\text {ext }}\right)$ are

$Q_{\text {scatt }}=\frac{\sigma_{\text {scatt }}}{\pi a^{2}}=\frac{2}{x^{2}} \sum_{n=1}^{\infty}(2 n+1)\left[\left|a_{n}\right|^{2}+\left|b_{n}\right|^{2}\right]$, and

$Q_{\mathrm{ext}}=\frac{\sigma_{\mathrm{ext}}}{\pi a^{2}}=\frac{2}{x^{2}} \sum_{n=1}^{\infty}(2 n+1) R_{e}\left(a_{n}+b_{n}\right)$,

where $\sigma_{\text {ext }}$ and $\sigma_{\text {scatt }}$ are the extinction and scattering crosssections respectively, $a$ is the grain radius, $x$ is the size parameter

\footnotetext{
1 http://www . met. tamu . edu/class/atmo689-1c/bhmie.pro
}

Table 2. Cloud condensates found in the atmosphere of solar system planets.

\begin{tabular}{cccc}
\hline \hline Condensate & Planet & $\begin{array}{c}\text { Condensation } \\
\text { temperature }^{+} \\
(\mathrm{K})\end{array}$ & $\begin{array}{c}\text { Pressure range }^{+} \\
\text {(cold-hot) } \\
(\text { bar })\end{array}$ \\
\hline $\mathrm{H}_{2} \mathrm{O}$ & $\mathrm{J}, \mathrm{S}, \mathrm{U}, \mathrm{N}$ & $274-348$ & $4.85-526$ \\
$\mathrm{NH}_{3}$ & $\mathrm{~J}, \mathrm{~S}, \mathrm{U}, \mathrm{N}$ & $147-163$ & $0.66-7.62$ \\
$\mathrm{NH}_{4} \mathrm{SH}$ & $\mathrm{J}, \mathrm{S}, \mathrm{U}, \mathrm{N}$ & $209-237$ & $2.01-42$ \\
$\mathrm{H}_{2} \mathrm{~S}$ & $\mathrm{~S}, \mathrm{U}, \mathrm{N}$ & $116-124$ & $0.66-3.23$ \\
$\mathrm{CH}_{4}$ & $\mathrm{U}, \mathrm{N}$ & $\sim 80$ & $0.94-1.2$ \\
\hline Titan tholins & Titan & $\leq 90$ & \\
\hline
\end{tabular}

Notes. ${ }^{(+)}$Carlson et al. (1988).

$(x=2 \pi a / \lambda)$, and $a_{n}$ and $b_{n}$ are the mix coefficients expressed in terms of the complex index of refraction (see Sharp \& Burrows 2007 for full equations). From this the extinction, scattering, and absorption cross section can be calculated as

$\sigma_{\text {ext }}=\sigma_{\text {scatt }}+\sigma_{\text {abs }}$.

\section{Transmission spectrum}

To compute the transmission spectrum for a given condensate we calculate the effective altitude, $z(\lambda)$, of the atmosphere as a function of wavelength using the analytical formula of Lecavelier Des Etangs et al. (2008) and the cross sections estimated from Mie scattering. This needs to be done separately 

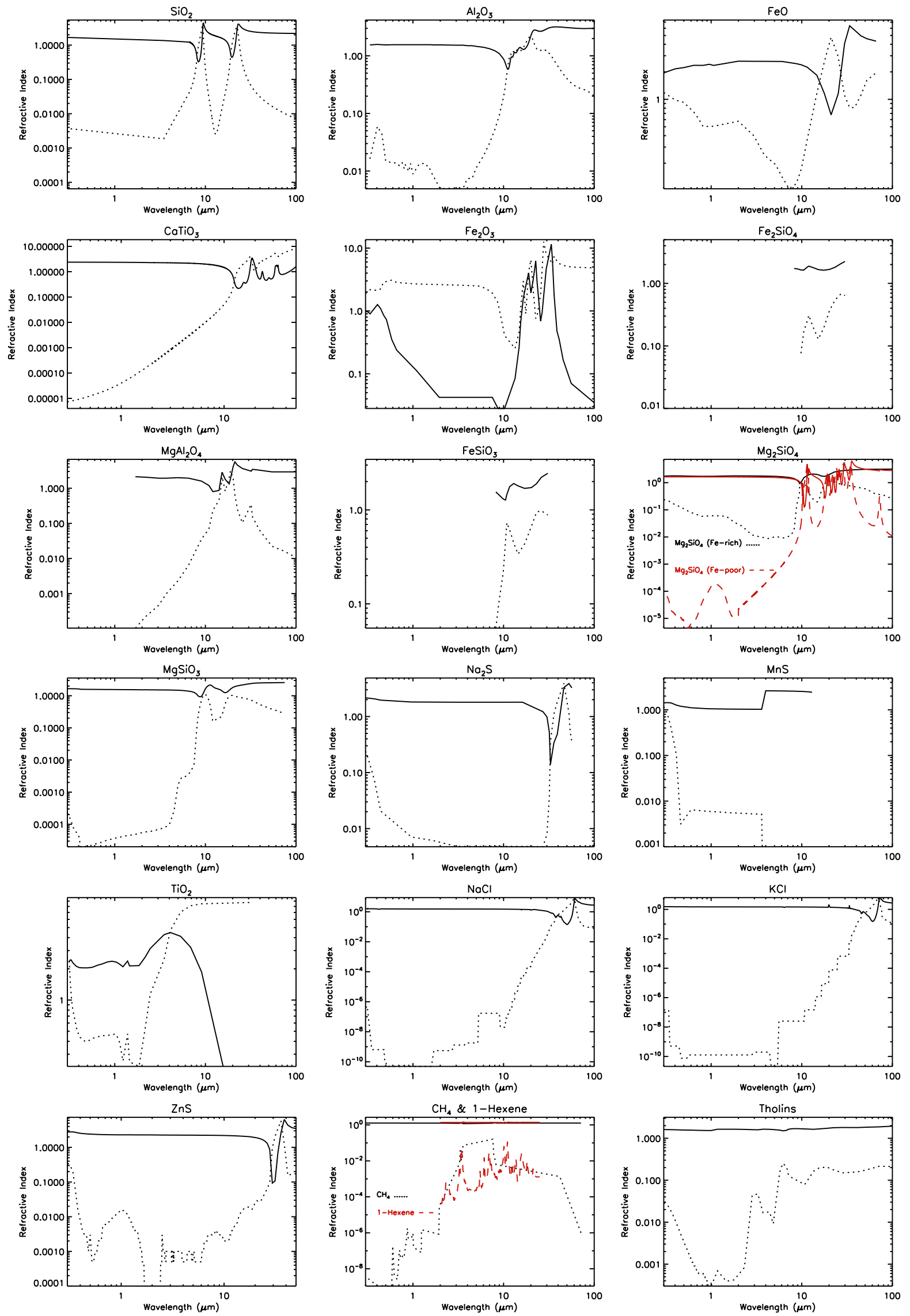

Fig. 1. Real (solid) and complex (dotted) index of refraction for each of the condensates listed in Table 1. Condensates are listed in terms of their condensation temperature from hot in the top left corner to cold in the bottom right corner. 
for each of the condensates considered as it relies upon the planetary scale height and specific abundance for each cloud considered. Here we use the well-studied hot Jupiter HD 189733b as an example atmosphere with $R_{\mathrm{p}}=1.138 R_{\mathrm{J}}, R_{*}=0.756 R_{\odot}$, $g_{\mathrm{p}}=3.34 \mathrm{~ms}^{-2}$, and $T_{\text {eff }}=1350 \mathrm{~K}$ (Southworth 2010; Torres et al. 2008). The effective altitude is given by

$z(\lambda)=H \ln \left(\frac{\xi_{\mathrm{abs}} P_{(z=0)} \sigma_{\mathrm{ext}}(\lambda)}{\tau_{\mathrm{eq}}} \times \sqrt{\frac{2 \pi R_{\mathrm{p}}}{k_{\mathrm{B}} T \mu g}}\right)$,

where $H$ is the planetary scale height, $\xi_{\text {abs }}$ is the abundance of the dominant species, $P_{z=0}$ is the reference pressure at $z=0, \sigma_{\text {ext }}(\lambda)$ is the wavelength dependent extinction cross-section, $\tau_{\text {eq }}$ is the equivalent optical depth at the measured transit radius $(\sim 0.56$; Lecavelier Des Etangs et al. 2008), and $k_{\mathrm{B}}$ is the Boltzmann constant.

A planetary scale height, $H$, is the altitude range over which the atmosphere pressure decreases by a factor of e, such that

$H=\frac{k_{\mathrm{B}} T}{\mu_{\mathrm{m}} m_{\mathrm{H}} g}$,

where $T$ is the estimated atmospheric temperature, $m_{\mathrm{H}}$ is the mass of a hydrogen atom, $\mu_{\mathrm{m}}$ is the mean molecular weight of the atmosphere, and $g$ is the surface gravity. For condensates their scale height, $H$, can potentially be smaller than the gaseous scale height; $H \sim H_{\mathrm{g}} / 3$ (Fortney 2005). Lecavelier Des Etangs et al. (2008) showed that, in the case of HD 189733b, the gaseous pressure scale height can be equated to that of the condensate scale height determined from the observed Rayleigh slope, which implies strong vertical mixing of condensates in the planetary atmosphere. We use the same assumption here. We compute the condensate cloud transmission spectra for an isothermal atmosphere. Following temperature-pressure profiles from Fig. 1 of Showman et al. (2008) and Fig. 5 of Fortney et al. (2010) it can be seen that at altitudes probed in slant geometry at the terminator, in the mbar pressure range, the transmission spectra are not overly sensitive to changes in temperature, where $\Delta T$ is of the order of $100 \mathrm{~K}$ (also see Howe \& Burrows 2012).

\subsection{Condensate abundance}

To determine the expected abundance of a certain condensate we first assume that it relies upon the metallicity abundance of the main atom from solar abundances (Burrows \& Sharp 1999). Here we use the example of $\mathrm{MgSiO}_{3}$ to demonstrate the calculation (Lecavelier Des Etangs et al. 2008),

$\xi_{\mathrm{MgSiO}_{3}}=\frac{3 a^{3} N_{\mathrm{A}} m_{\mathrm{p}} \mu_{\mathrm{MgSiO}_{3}} \xi_{\mathrm{Mg}}}{2 \pi \rho_{\mathrm{MgSiO}_{3}}}$

where $\xi_{\mathrm{Mg}}$ is the solar abundance of $\mathrm{Mg}, \rho_{\mathrm{MgSiO}_{3}}$ is the density of $\mathrm{MgSiO}_{3}$, and $N_{\mathrm{A}} m_{\mathrm{p}}$ is Avogadro's constant multiplied by the mass of a proton acting as a scaling factor.

The effective altitude, $z(\lambda)$, is then added to the bulk planetary radius to compute the observable transmission spectrum, $R_{\mathrm{p}}(\lambda) / R_{*}$, of the condensate.

Figure 2 shows the transmission spectrum for each cloud condensate in Table 1 computed for the atmosphere of HD $189733 b$ given a condensate grain size of $0.1 \mu \mathrm{m}$ and abundances $1 \times$ solar. We plot the spectrum in units of scale height, $H$, such that the transmission spectra of different exoplanets will appear very similar, as $z / H$ is only weakly dependent upon specific values of $R_{\mathrm{p}}$ or $g$. The different condensates have been separated into groups of their primary diatomic bond to highlight
Table 3. Vibrational modes for the major diatomic bond species in the different cloud condensates considered in this paper.

\begin{tabular}{cccc}
\hline \hline $\begin{array}{c}\text { Major } \\
\text { bond }\end{array}$ & $\begin{array}{c}\text { Reduced } \\
\text { mass, } \mu_{M} \\
(\mathrm{~g})\end{array}$ & $\begin{array}{c}\text { Vibrational } \\
\text { frequency, } v \\
\left(\mathrm{~cm}^{-1}\right)\end{array}$ & $\begin{array}{c}\text { Wavelength, } \\
\lambda \\
(\mu \mathrm{m})\end{array}$ \\
\hline $\mathrm{Si}-\mathrm{O}$ & 10.192 & $1110-830^{a}$ & $9-12$ \\
$\mathrm{Al}-\mathrm{O}$ & 10.043 & $1100-350^{c}$ & $9-28.7$ \\
$\mathrm{Fe}-\mathrm{O}$ & 12.436 & $790^{b}$ & 12.5 \\
$\mathrm{Ti}-\mathrm{O}$ & 11.99 & $850-150^{d}$ & $16-66$ \\
$\mathrm{MnS}$ & 20.247 & $295-220^{e}$ & 20.2 \\
$\mathrm{ZnS}$ & 21.51 & $464^{f}$ & 21.5 \\
$\mathrm{NaCl}$ & 13.95 & $366^{g}$ & 13.95 \\
$\mathrm{KCl}$ & 18.60 & $281^{g}$ & 18.6 \\
$\mathrm{C}-\mathrm{H}$ & 0.923 & $3032^{a}$ & 3.3 \\
\hline
\end{tabular}

Notes. ${ }^{(a)}$ Glassgold \& Graham (2008); ${ }^{(b)}$ Lehnert et al. (2002); ${ }^{(c)}$ Saniger (1995); ${ }^{(d)}$ Gillet et al. (1993); ${ }^{(e)}$ Batsanov \& Derbeneva (1969); ${ }^{(f)}$ Kröger \& Meyer (1954); ${ }^{(g)}$ Rice \& Klemperer (2004).

the similarities between different condensate spectra when the absorption is dominated by one vibrational state.

\subsection{Vibrational modes}

Major dust spectral features are determined by vibrational modes. Silicate dust has a major feature at $10 \mu \mathrm{m}$, while hydrocarbons have major features at $3 \mu \mathrm{m}$. The vibrational frequency, $v$, for the major dipole bonds considered here can be estimated assuming a harmonic oscillation with,

$v=\frac{1}{2 \pi c} \sqrt{\frac{K}{\mu_{M}}}$

where $c$ is the speed of light, $K$ is the force constant of the bond considered, and $\mu_{M}$ is the reduced mass in grams $\mu_{M}=$ $\left(m_{1} m_{2}\right) /\left(m_{1}+m_{2}\right)$.

The calculated vibrational modes and their corresponding wavelength ranges for each of the major bond species considered in this paper can be seen in Table 3. Unlike gaseous molecules, where the rotational structure of the molecules can be observed as individual narrow absorption lines, solid molecules suppress the rotational structure as they cannot rotate freely resulting in a smearing of the absorption lines into broad peaks. Figure 2 displays the transmission spectra calculated using Mie theory, and the commonalities between different condensates with the same major vibrational modes due to the main diatomic bond can be seen.

For simple diatomic molecules like $\mathrm{MnS}$ and $\mathrm{ZnS}$ or $\mathrm{NaCl}$ and $\mathrm{KCl}$ the slight difference in vibrational modes can be seen clearly in the sharp absorption features of their transmission spectra. More complex molecules show broad absorption features across a range of wavelengths centred around the vibrational wavelength of the major dipole.

\subsection{Particle size and distribution}

The transmission spectrum for all condensate species is highly dependent on the size $(a)$ of the particles composing the cloud. To loft particles to the upper atmosphere, the expectations of vertical mixing need to be taken into account. Models from Parmentier et al. (2013) and Heng \& Demory (2013) show that strong vertical mixing can keep micron or sub-micron sized particles aloft in the atmosphere where grain sizes between 0.001 

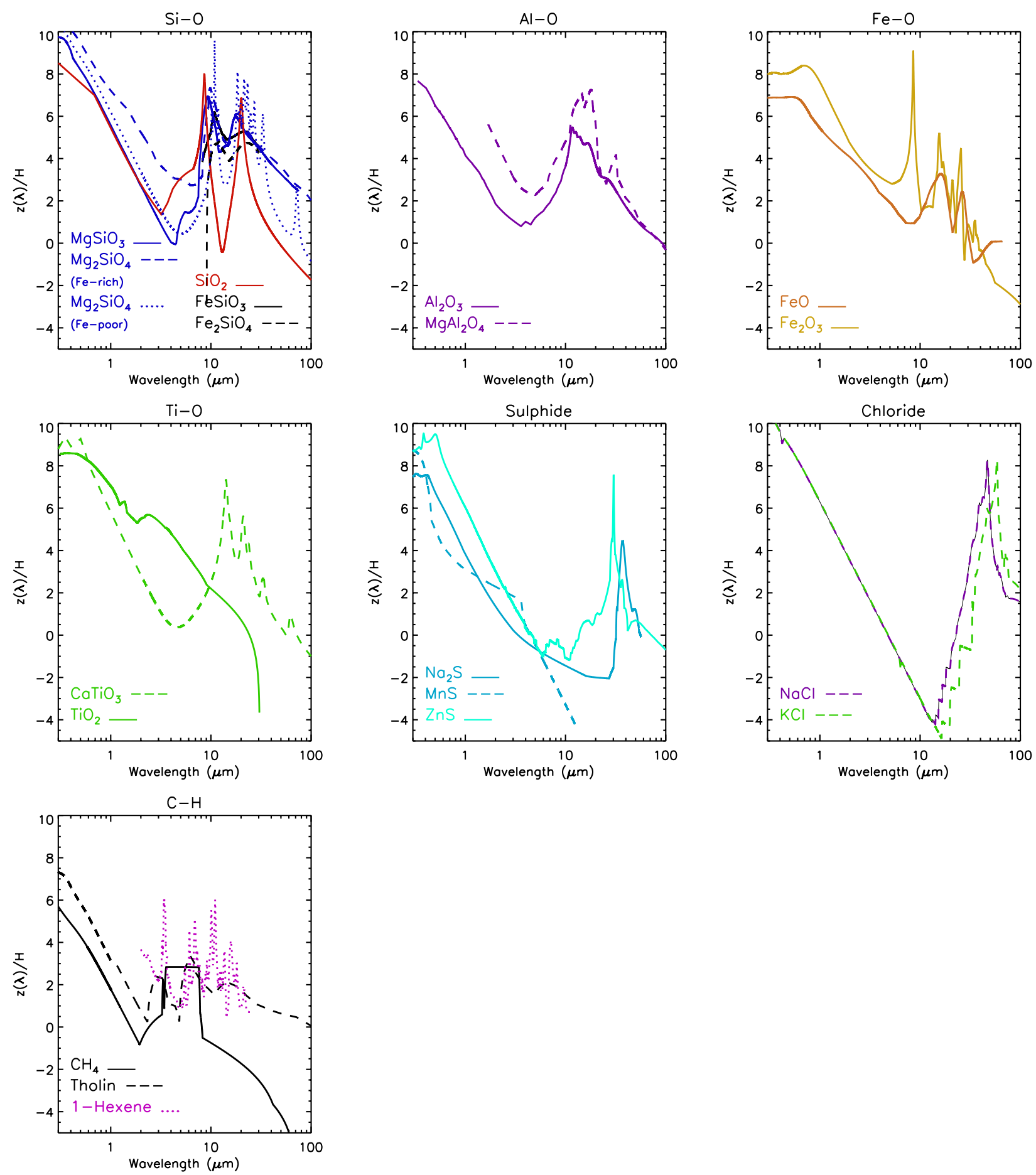

Fig. 2. Transmission spectra for a number of condensates expected in hot Jupiter atmospheres using HD $189733 \mathrm{~b}$ system parameters $(a=0.1 \mu \mathrm{m}$, $\left.T_{\text {eff }}=1350 \mathrm{~K}\right)$. The condensates have been separated out according to their primary bond where vibrational modes between these species dominate the spectra.

and $100 \mu \mathrm{m}$ were considered. Lecavelier Des Etangs et al. (2008) show that in the Rayleigh regime, the cross section is proportional to $a^{6}$. This makes the scattering and resulting transmission spectrum largely dependent on the largest grain size in the particle distribution of the cloud.

To demonstrate the effect of larger grain sizes in clouds with particle distributions, we applied a series of log-normal grain size distributions to our cloud particles and calculated the resulting transmission spectrum. Log-normal distributions (ln $N(\mu, \sigma))$ are dependent upon the centre $(\mu)$ of the distribution and the width $(\sigma)$. We set a grid of log-normal distributions with $\mu=0.001-7.5 \mu \mathrm{m}$ and $\sigma=0.05-1.0$ and computed the cumulative transmission spectrum for the condensate clouds of each distribution. Figures 3-5 show three distributions with the contributing grain sizes for the transmission spectra, and the resulting cumulative spectrum. These figures demonstrate the effect of larger grain sizes on the cumulative transmission spectrum, where a small number of large grain size particles dominates the 
H. R. Wakeford and D. K. Sing: Transmission spectral properties of clouds for hot Jupiter exoplanets

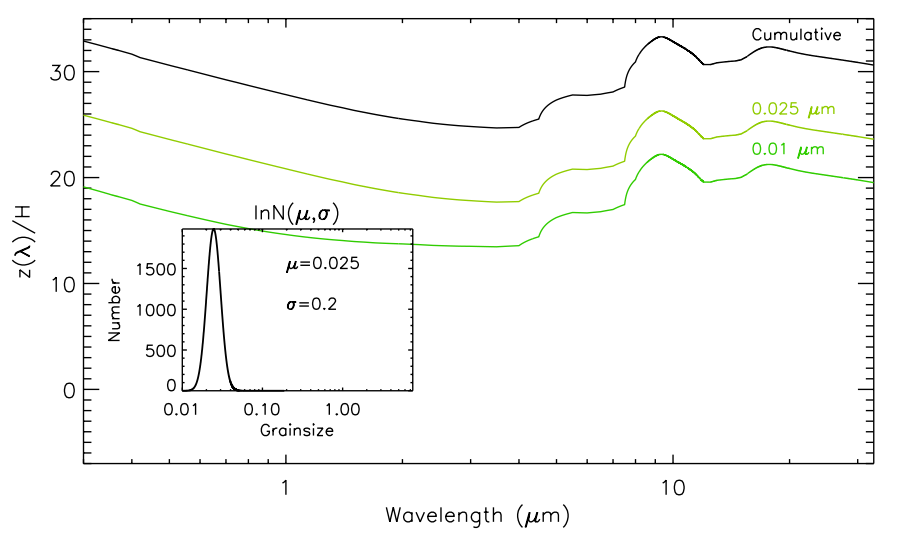

Fig. 3. Cumulative transmission spectrum of a log-normal distribution centered at $0.025 \mu \mathrm{m}$ with a width of 0.2 showing each of the individual spectra contributing to the final transmission spectra of the cloud. The sub-plot shows the distribution used for this cloud with the $x$-axis on a log scale.

resulting cloud spectra. For distributions with only sub-micron sized particles, vibrational mode absorption features in the infrared can be seen. As evident in Figs. 3-5 all the cumulative distribution transmission spectra can be well approximated by a single grain size which is the largest in the distribution, as expected given the $\sigma \propto a^{6}$ relation. Distributions with sizes larger than $\sim 1 \mu \mathrm{m}$ tend toward completely flat, featureless spectra in the optical and infrared. Figure 5 shows the transition from prominent absorption features visible in sub-micron sized particle spectra to flat spectra where the grain sizes become larger than $\sim 1 \mu \mathrm{m}$.

Key features in hot Jupiter transmission spectra can be used as diagnostic tools to set limits on the grain size of observed clouds, and predict the likelihood of features being observed at longer wavelengths. The presence of a Rayleigh slope in the optical part of the spectra, like that seen in HD 189733b (Pont et al. 2013), indicates that any clouds present in the atmosphere at transmission spectral altitudes are likely made of submicron sized grain particles. Further evidence of infrared features (McCullough et al. 2014), along with the general planetary parameters, can be used to diagnose the probability of observing condensate absorption features in the mid infrared where a majority of the condensate vibrational modes are observed. However, if larger grain sizes are lofted up in the atmosphere to altitudes probed by transmission spectra it is unlikely significant optical to infrared features will be observed, such as in the flat transmission spectra of HAT-P-32b (Gibson et al. 2013).

\section{Discussion}

A majority of current exoplanet spectra are constructed from ground-based measurements with additional low resolution spectra from HST at wavelengths short of $1.7 \mu \mathrm{m}$, and in the infrared from Spitzer (e.g. Gibson et al. 2013; Pont et al. 2013; Sing et al. 2013; Nascimbeni et al. 2013). At present, these observations focus on the optical and near-infrared regions of the spectrum, revealing information on the portion of transmission spectra for aerosols where only scattering features are seen (e.g. Pont et al. 2013; Sing et al. 2013; see Fig. 7). We have used Mie theory, and the expectations of current cloud modelling, to compute approximate hot Jupiter transmission spectra from the optical to far infrared regime. When interpreting observations, the slope of spectra in the optical regime is proportional to the temperature of the atmosphere and can be indicative of specific

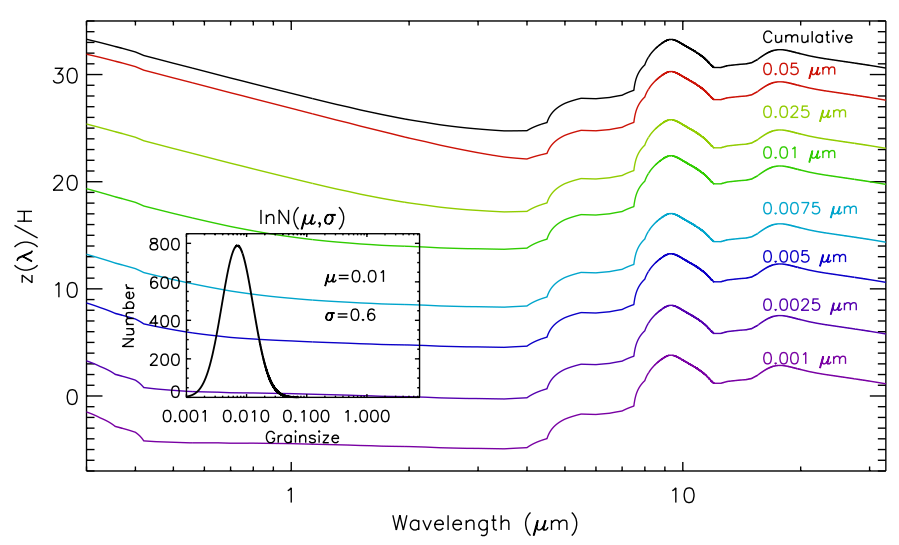

Fig. 4. Cumulative transmission spectrum of a log-normal distribution centered at $0.01 \mu \mathrm{m}$ with a width of 0.6 showing each of the individual spectra contributing to the final transmission spectra of the cloud. The sub-plot shows the distribution used for this cloud with the $x$-axis on a log scale.

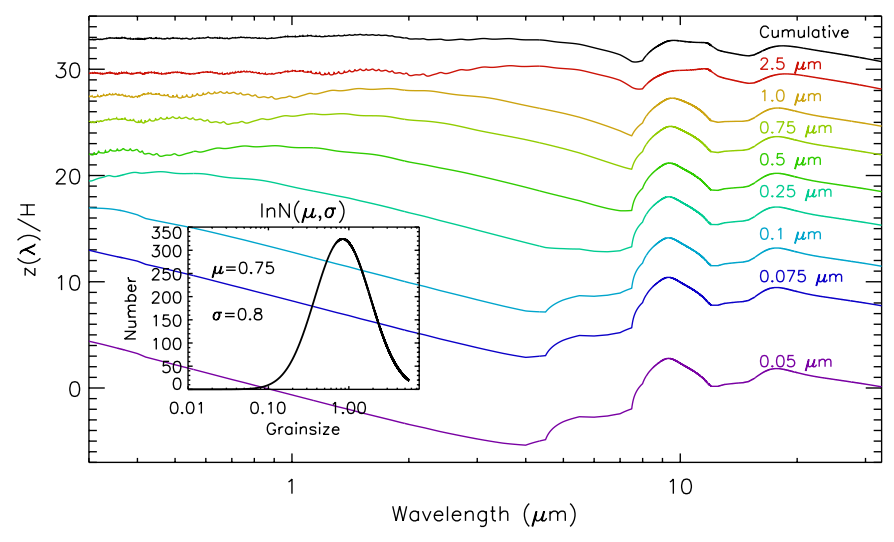

Fig. 5. Cumulative transmission spectrum of a log-normal distribution centered at $0.75 \mu \mathrm{m}$ with a width of 0.8 showing each of the individual spectra contributing to the final transmission spectra of the cloud. The sub-plot shows the distribution used for this cloud with the $x$-axis on a $\log$ scale.

species when small grain sizes are considered. Additionally, absorption features in the near- and mid-infrared spectra can be identified as the vibrational modes of the major bond pair in the condensates considered, providing composition information.

Models of brown dwarf atmospheres suggest that silicates are likely to form the dominant cloud structures in hot Jupiter atmospheres where temperatures are greater than $\sim 1000 \mathrm{~K}$, with sulphide clouds becoming dominant at temperatures below $900 \mathrm{~K}$ at which chlorides also begin to condense out (Morley et al. 2012). It is expected that hotter atmospheres could have a greater abundance of $\mathrm{Al}_{2} \mathrm{O}_{3}$ and $\mathrm{Fe}_{2} \mathrm{O}_{3}$, as silicates will not have condensed out. Additionally, $\mathrm{Al}_{2} \mathrm{O}_{3}$ and $\mathrm{CaTiO}_{3}$ will not be present at the mbar pressure level in atmospheres with $T_{\text {eff }} \leq$ $1600 \mathrm{~K}$ as $\mathrm{Al}$ and $\mathrm{Ca}$ get locked up in magnesium oxides in deeper layers of the atmosphere.

This work shows that while it is unlikely that we will be able to distinguish between individual silicate dust species, similar to the ISM and brown dwarfs, we may be able to discern a contrast between separate dust sub-classes like those shown in Fig. 2. As a result, an observational distinction can be placed on photochemically generated species, such as hydrocarbons with a dominant $\mathrm{C}-\mathrm{H}$ bond, and condensation chemistry produced molecules like those with a dominant $\mathrm{Si}-\mathrm{O}$ bond. Along with 
wavelength differentiation between major species' vibrational modes, there is also a significant altitude distinction in the transmission spectra where features in the near infrared can extend above the optical slope. At these wavelengths, predominant cloud absorption features could compete with $\mathrm{H}_{2} \mathrm{O}$ and other molecules in the near infrared, potentially obscuring expected atmospheric features.

\subsection{Interpreting hot Jupiter transmission spectra}

Presently exoplanet spectra are limited to the optical and nearinfrared regime below $1.7 \mu \mathrm{m}$ with intermittent wavelength coverage into the infrared with Spitzer. Current exoplanet broadband transmission spectra that have evidence for clouds show commonalities in the optical regime, where all scatterers appear to be very similar. There is growing evidence for differences in their molecular transmission spectral signatures. For instance, WFC3 spectra have detected large $\mathrm{H}_{2} \mathrm{O}$ features in HAT-P-1b and WASP-19b (Wakeford et al. 2013; Huitson et al. 2013) while these features can be muted or even absent for other planets like WASP-12b and WASP-31b (Sing et al. 2013, 2014).

The hot Jupiter HD 189733b has been extensively studied into the infrared with observations by cold Spitzer at 3.6, 4.5, 5.8, 8.0, and $24 \mu \mathrm{m}$ (Knutson et al. 2007, 2012). Measurements of HD $189733 \mathrm{~b}$ in the infrared hint at the presence of molecular absorption by water in the planet's upper atmosphere with additional evidence from both high resolution spectroscopy and eclipse spectral data (Grillmair et al. 2008; Birkby et al. 2013; Pont et al. 2013; McCullough et al. 2014). We use the HD 189733b transmission spectrum as an example hot Jupiter atmosphere, and discuss potential spectral features with regards to the condensates and photochemical species shown in this paper. Absorption from gaseous species such as $\mathrm{H}_{2} \mathrm{O}, \mathrm{CO}$, and $\mathrm{CH}_{4}$ can be present in the infrared and obscure condensate features. For HD 189733b, optical scattering can be seen to high altitudes spanning $\sim 7 \mathrm{H}$ with infrared data giving strong constraints on the altitude levels of the gaseous molecular species such as $\mathrm{H}_{2} \mathrm{O}$. While only a subset of the condensates considered here are appropriate for HD $189733 \mathrm{~b}$, given the planetary parameters, we use it to illustrate where other condensates could be detectable in hotter or cooler exoplanets, given their vibrational modes.

Using the HD 189733b transmission spectra as an example hot Jupiter we address two questions; what transmission spectral features for the various condensates can we observe and at what wavelength are we likely to observe them?

\subsubsection{Grainsize}

We fit the HD 189733b transmission spectrum with a grid of log-normal distributions applied to the condensate $\mathrm{MgSiO}_{3}$. We fit the cumulative spectrum of each grid point with the complete HD 189733 b spectrum by allowing only the altitude to vary. Figure 6 shows the $\Delta \chi^{2}$ fit for each log-normal particle distribution, where the position of the expanded distributions in Figs. 3-5 are labeled A, B, and C respectively. The best fit distribution is shown to be $0.025 \mu \mathrm{m}$ when $\sigma$ is small, with wider distributions where the maximum grain size is $0.025 \mu \mathrm{m}$ also providing good fits to the data.

Using the best-fitting condensate model we can rule out the presence of particles larger than $\sim 0.025 \mu \mathrm{m}$ in the atmosphere of HD 189733b, which would generate a "flat" spectrum from the optical through the infrared and likely hide the deeper $\mathrm{H}_{2} \mathrm{O}$ features observed in the near infrared. Expanding these results

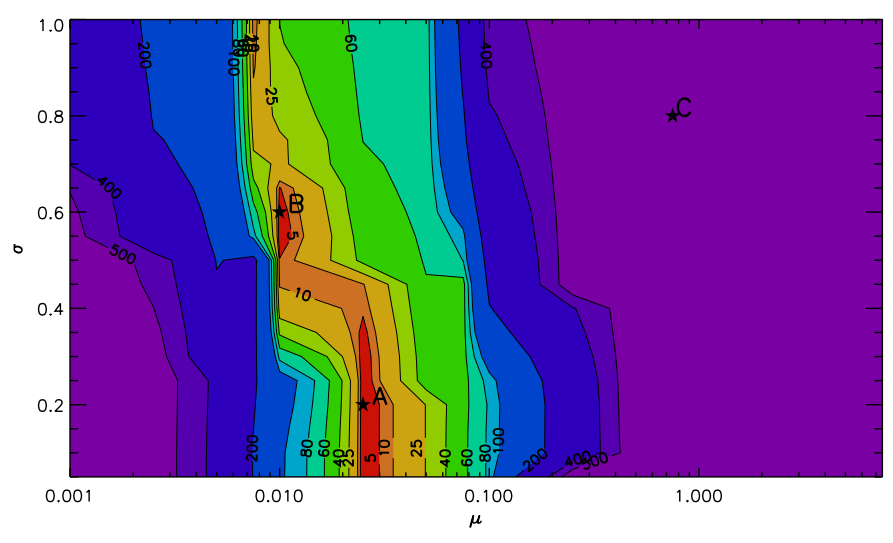

Fig. 6. $\Delta \chi^{2}$ grid for a series of log-normal grainsize distributions when fitted to the HD $189733 \mathrm{~b}$ transmission spectral data. The best fit occurs for distributions which contain $\sim 0.025 \mu \mathrm{m}$ size particles. The distributions in Figs. 3-5 are labeled A, B, and C respectively.

to other hot Jupiters, a similar exploration of the grain size distributions which are compatible with optical and near-infrared transmission spectra can be used to predict potential condensate vibrational mode features in the infrared. These findings suggest such infrared vibrational modes will only be observable in transmission spectra where optical scattering is present with submicron size particle distributions.

Given the largest grain-sized particle in the distribution produces a reasonable approximation of the transmission spectrum's shape, as shown by Figs. 3-6, for simplicity a single grain size is assumed in subsequent sections.

\subsubsection{Condensate spectra}

We fit single grain size condensate cloud spectra from $0.001-10 \mu \mathrm{m}$ calculated using Mie theory for each of the condensates in Table 1 to the HD $189733 \mathrm{~b}$ data from $0.3-1.0 \mu \mathrm{m}$ of Pont et al. (2013) where significant Rayleigh scattering is observed.

Figure 7 shows the observed HD $189733 \mathrm{~b}$ transmission spectrum from 0.3 to $24 \mu \mathrm{m}$ (Pont et al. 2013; McCullough et al. 2014) with a representative condensate spectrum for each of the major diatomic vibrational modes shown in Fig. 2. By allowing only the altitude to vary, we find a best-fit grain size of $0.025 \mu \mathrm{m}$ for each of the condensates matching the grain size distribution fits shown in Fig. 6.

The cloud model spectra shown in Fig. 7 demonstrate that while scattering by aerosols becomes negligible at longer ( $\sim 2 \mu \mathrm{m})$ wavelengths, as the cross-section becomes small, significant infrared absorption features appear. In some cases, these vibrational absorption features are seen to rise to altitudes in the transmission spectra above the optical scattering levels, making them potentially detectable. Additionally, a number of the absorption features span multiple scale heights becoming visible above the expected molecular bands of the abundant gaseous species $\left(\mathrm{H}_{2} \mathrm{O}, \mathrm{CH}_{4}\right)$. We use a Fortney et al. (2010) model of HD 189733 b which has a $1000 \times$ enhanced scattering to estimate the amplitude and relative location of molecular features in the transmission spectrum, fitting for the HD 189733b transmission data (Pont et al. 2013; McCullough et al. 2014) by allowing only the model altitude to vary. The Fortney et al. (2010) model is a good fit to the optical slope, the $1.4 \mu \mathrm{m} \mathrm{H}_{2} \mathrm{O}$ feature, and the Spitzer data, though only contains an artificial pure-Rayleigh scattering component, which has no effect on the transmission 
H. R. Wakeford and D. K. Sing: Transmission spectral properties of clouds for hot Jupiter exoplanets

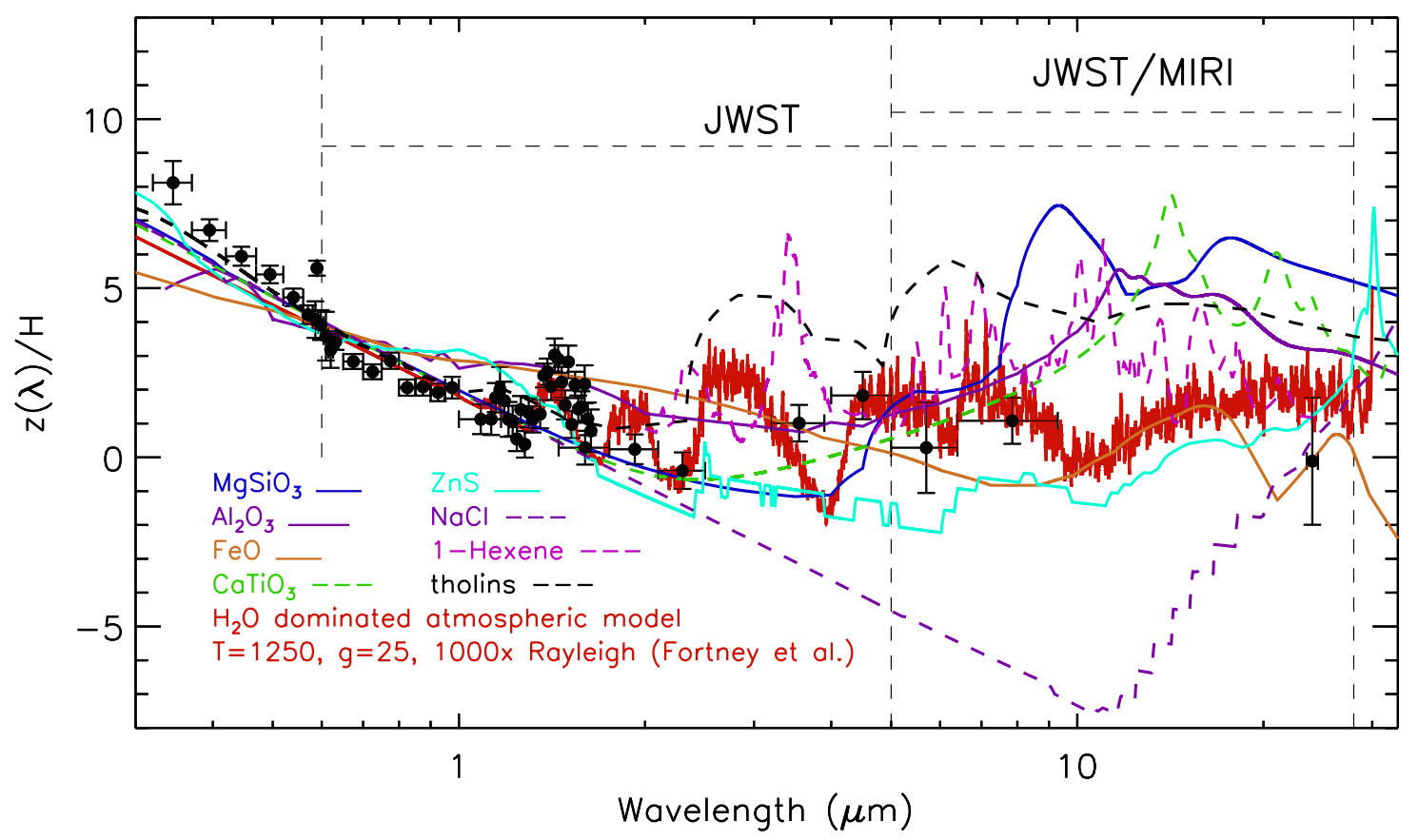

Fig. 7. Transmission spectrum of HD 189733b (Pont et al. 2013; McCullough et al. 2014) compared to a number of different condensates with a best fit grainsize of $0.025 \mu \mathrm{m}$ and $\mathrm{a}_{2} \mathrm{O}$ dominated atmospheric model from Fortney et al.

spectrum longer than $\sim 2 \mu \mathrm{m}$. As evident in Figs. 2 and 7 , the scattering properties of each condensate considered here are expected to be similar shortward of $3 \mu \mathrm{m}$, as absorption features, due to the vibrational modes of the molecules, are not observed in the optical and near infrared (see Table 3). Here we consider each of the major bond species in turn and discuss the absorption features which may be detectable for sub-micron size grains.

$\mathrm{Si}-\mathrm{O}$ : each of the silicates considered to be present in the atmospheres of hot Jupiters show a strong Rayleigh slope in the optical up to $3 \mu \mathrm{m}$ with an exception of Fe-rich $\mathrm{Mg}_{2} \mathrm{SiO}_{4}$ which shows additional absorption to that of standard Rayleigh. Major vibrational mode absorption features emerge from $9-12 \mu \mathrm{m}$ spanning multiple scale heights matching the altitude at the top of the optical Rayleigh slope. In Fig. 7 these features are represented by the transmission spectrum of $\mathrm{MgSiO}_{3}$ which has an absorption feature across several microns reaching above the $1000 \times$ Fortney spectra. If silicate clouds dominated by small particle sizes are present in hot Jupiter atmospheres, it is likely that their presence and pressure altitude can be determined by transmission spectral features observed in the infrared by JWST/MIRI.

Al-O: aluminium oxides also show distinct Rayleigh properties in the optical, with broadband absorption features extending from $9-28 \mu \mathrm{m}$. While it is unlikely that the atmosphere of HD 189733 b contains Al-O condensate clouds, it is possible that hotter Jupiters, such as WASP-12b which has a significant optical slope, can show Al-O condensate features. As evident in Fig. 7, the peak of the Al-O vibrational mode feature forms a broadband absorption spectrum between that of the two silicate absorption features at $10-12 \mu \mathrm{m}$. It is possible that if $\mathrm{Si}-\mathrm{O}$ or $\mathrm{Al}-\mathrm{O}$ condensate clouds are present in a hot Jupiter atmosphere, such as the high temperature condensates $\mathrm{SiO}_{2}$ or $\mathrm{Al}_{2} \mathrm{O}_{3}$, either could be identified where the altitude of the obscuring feature can exceed that of potential gaseous molecules from $\sim 8-20 \mu \mathrm{m}$.

$\mathrm{Fe}-\mathrm{O}$ : the major vibrational mode features of iron oxides are centred at $\sim 14$ with both broad- and narrow-band features from $\sim 9-40 \mu \mathrm{m}$. For small grain sizes like that shown in Fig. 7 the transmission spectrum of $\mathrm{Fe}-\mathrm{O}$ compounds exhibit a flattened slope from $0.3-2 \mu \mathrm{m}$ which could obscure the expected molecular water bands at 1.1 and $1.4 \mu \mathrm{m}$. While potential iron-oxide narrow-band features, like those of $\mathrm{Fe}_{2} \mathrm{O}_{3}$, may be visible above the expected $\mathrm{H}_{2} \mathrm{O}$ dominated transmission spectra (see Fig. 2), it is unlikely that broadband features will be observed at the mbar pressure level where spectra dominated by gaseous vibrorotational bands are several scale heights above any potential absorption features.

Ti-O: Fig. 7 shows the computed transmission spectrum of $\mathrm{CaTiO}_{3}$, selected to represent potential Ti-O condensate clouds formed in hot Jupiter atmospheres. Ti-O molecules have vibrational mode features from $16-66 \mu \mathrm{m}$ which is partially covered by JWST/MIRI. While the cooler $\mathrm{TiO}_{2}$ condensate shows little to no distinct features (see Fig. 2), the hotter condensate $\mathrm{CaTiO}_{3}$ has visible narrow absorption feature centred at $\sim 15 \mu \mathrm{m}$ and $\sim 21 \mu \mathrm{m}$, which span several scale heights above the $1000 \times$ Fortney model.

Sulphides: sulphur-bearing compounds are expected to condense in hot Jupiter atmospheres at $\sim 1100 \mathrm{~K}$ with highly scattering properties shortward of $\sim 10 \mu \mathrm{m}$. Similar to iron oxide condensates, the transmission spectra shows a leveling off between 0.5 and $1 \mu \mathrm{m}$ which can potentially be used to help identify such clouds in UV and optical transmission spectra. In addition, the hotter condensates such as $\mathrm{Al}-\mathrm{O}-$ and $\mathrm{Fe}-\mathrm{O}$-bearing compounds are not expected to coincide with sulphide condensate clouds, which helps identification. However, any additional features distinct to sulphur-bearing compounds do not emerge above the $\mathrm{H}_{2} \mathrm{O}$ dominated model in hot Jupiter transmission spectra within the wavelength limits of JWST.

Chlorides: Alkali chlorides begin to condense at around $800 \mathrm{~K}$, depleting the expected $\mathrm{Na}$ and $\mathrm{K}$ atomic species in the planets upper atmosphere. Figures 2 and 7 show that these condensates are highly scattering well into the infrared following a Rayleigh slope to $\sim 10 \mu \mathrm{m}$. Vibrational modes are unlikely 


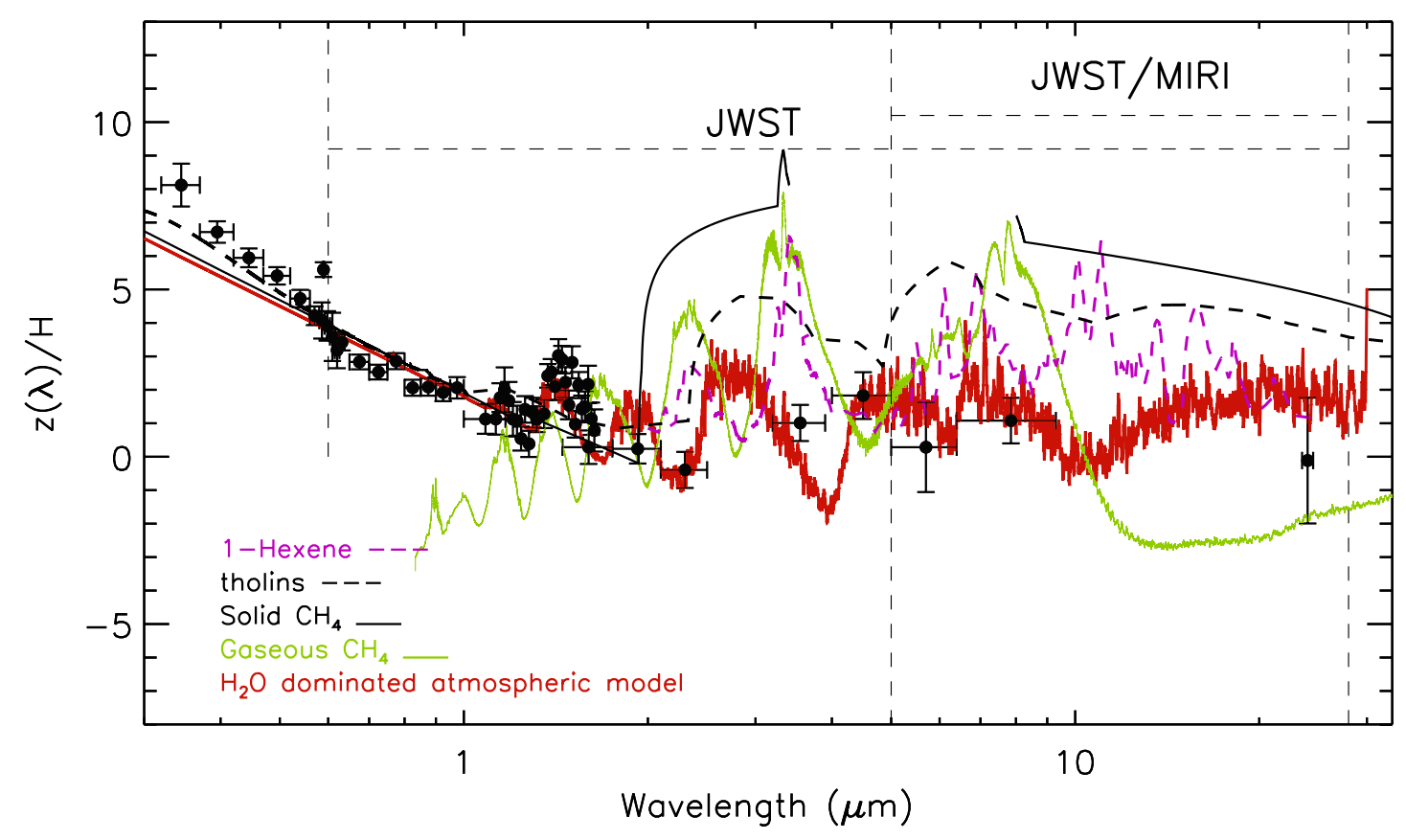

Fig. 8. Transmission spectrum of HD 189733 b (Pont et al. 2013; McCullough et al. 2014) compared to hydrocarbon condensates and gaseous $\mathrm{CH}_{4}$ (Yurchenko \& Tennyson 2014; Amundsen et al. 2014) and $\mathrm{C}_{2} \mathrm{H}_{4}$ (Rothman et al. 2009; Sharp \& Burrows 2007).

to be observed in hot Jupiter transmission spectra due to the obscuring molecular features, similar to sulphur-bearing condensate species. However, there is potential for chloride clouds to be inferred in cooler exoplanetary atmospheres where strong Rayleigh scattering is observed in the optical and there is no evidence for atomic gaseous $\mathrm{Na}$ or $\mathrm{K}$ in the optical spectra, suggesting that the species may have condensed out of the atmosphere forming clouds of liquid or solid particles.

Spectral measurements well into the infrared with extended wavelength resolution have the potential to differentiate between condensate species for a wide range of exoplanetary atmospheres as most of the cloud species have their lowest opacities at these mid-infrared wavelength regions. The $24 \mu \mathrm{m}$ point, of HD 189733b further aids in the interpretation of the planets atmosphere placing strong constraints on the particle size given the optical vs. infrared absorption level, also noted by Lee et al. (2014). While these vibrational modes of major bond species can be used to help identify the condensate cloud in the exoplanetary atmosphere, it is hard to ascertain the specific condensate responsible for the absorption feature present. Figure 7 shows cloud spectra extending above that of gaseous species, with a majority of potentially identifiable species having one or more absorption features in the wavelength range covered by JWST.

Identifying the different vibrational modes of potential cloud condensates and constraining/comparing the species in different exoplanet atmospheres could provide valuable insight into condensation chemistry over large temperature ranges. While the current broadband photometry is not of sufficient spectral resolution to distinguish between the models considered here, the situation should rapidly change with JWST.

\subsubsection{Photochemical vs. Condensation}

Although unlikely to be present in the atmosphere of HD 189733b, we have also included clouds composed of hydrocarbon species, such as hexene and Titan tholins, which may be generated photochemically. It can be seen that, although a poor fit to the HD $189733 \mathrm{~b}$ data, the major absorption features for condensate hydrocarbons extend above the $1000 \times$ Fortney model at the $\sim 3 \mu \mathrm{m}$ wavelength of the $\mathrm{C}-\mathrm{H}$ vibrational mode and thus may be potentially observed for other planets.

In cooler planetary atmospheres, photochemistry is expected to play a key role in the overall cloud composition in the generation of gaseous hydrocarbons. When the planetary $\mathrm{C} / \mathrm{O}$ ratio is greater than 1, the abundance of carbon-bearing compounds increases significantly, with disequilibrium processes enhancing their abundance over that of other species (Moses et al. 2013). Figure 8 shows the transmission spectrum of HD 189733b with the considered hydrocarbon species as well as the expected transmission spectrum for gaseous $\mathrm{CH}_{4}$ at solar abundance. The opacities for gaseous $\mathrm{CH}_{4}$ are calculated from the new ExoMol line list (Yurchenko \& Tennyson 2014), with the line width parameters as in Amundsen et al. (2014). It can be seen that the gaseous $\mathrm{CH}_{4}$ transmission spectral features overlap considerably with that of the hydrocarbon condensate cloud spectra, specifically at the $3 \mu \mathrm{m}$ range where the vibrational mode of the $\mathrm{C}-\mathrm{H}$ bond is responsible for the absorption feature. There are, however, still some notable differences which may be used to differentiate between the two states with additional absorption features shown in both Hexene $(\sim 10 \mu \mathrm{m})$ and Tholin $(\sim 6 \mu \mathrm{m})$ condensate spectra which emerge several scale heights above both the $\mathrm{H}_{2} \mathrm{O}$ dominated exoplanet spectra and that of the photochemically generated gaseous $\mathrm{CH}_{4}$ (see Fig. 8).

\subsection{James Webb Space Telescope}

We have considered the different condensates that are expected to form condensate clouds in hot Jupiter exoplanet atmospheres with specific consideration to the wavelengths covered by James Webb Space Telescope (JWST). JWST, set to launch in October 2018, is a NASA mission, with significant contributions from both ESA and CSA. JWST is a $6.5 \mathrm{~m}$ near- to midinfrared telescope that will orbit at the Sun-Earth L2 point giving it an uninterrupted view of the sky. JWST is equipped with low, 
H. R. Wakeford and D. K. Sing: Transmission spectral properties of clouds for hot Jupiter exoplanets

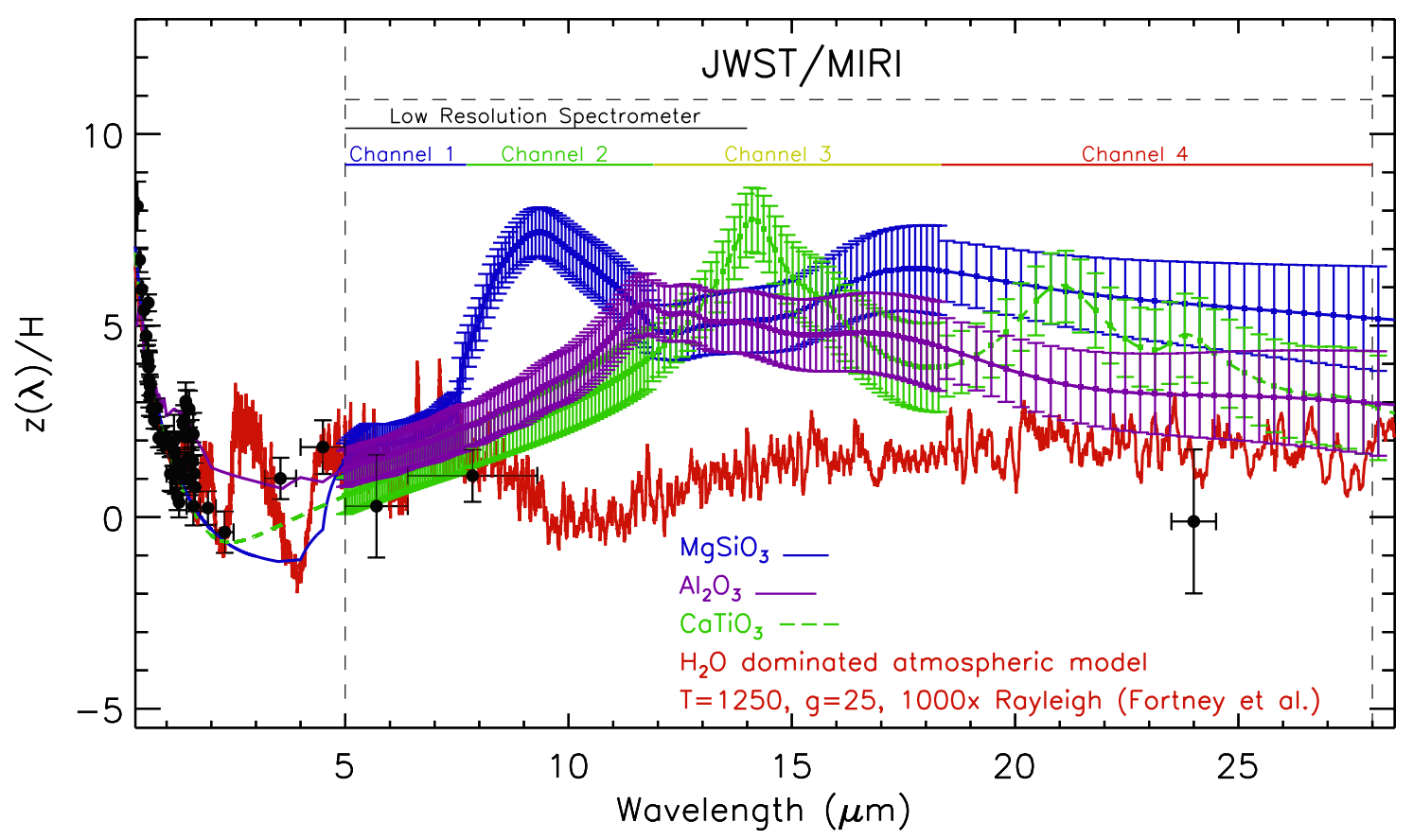

Fig. 9. Transmission spectrum of HD 189733 b (Pont et al. 2013; McCullough et al. 2014) over-plotted on cloud model spectra set to $R \sim 50$ for JWST/MIRI Medium resolution spectrograph channels 1-3 and $R \sim 30$ for channel 4 with the wavelength coverage highlighted above the different regions of the spectra they cover. We also highlight the region of the spectrum covered by the low resolution spectrometer $(5-14 \mu \mathrm{m})$.

medium, and high resolution spectrographs from the instruments NIRSPEC $(0.6-5 \mu \mathrm{m})$ and MIRI $(5-28 \mu \mathrm{m})$.

MIRI detectors are similar to Spitzer IRAC 5.8 and $8.0 \mu \mathrm{m}$ with an expected noise floor less than $100 \mathrm{ppm}$. It is the only JWST instrument that will observe wavelengths greater than $5 \mu \mathrm{m}$. The medium resolution spectrometer (MRS) is composed of four channels from 5-28 $\mu \mathrm{m}$ with a resolution $\sim 3000-1000$. To obtain a full transmission spectrum with MRS from 5-28 $\mu \mathrm{m}$ four separate transit observations are required to observe in all four channels equaling $\sim 24 \mathrm{~h}$ of observations for a majority of known exoplanet targets.

Here we use HD 189733b as an example hot Jupiter to simulate the transmission spectrum of our cloud condensate models and interpret the results with respect to the estimated preci$\operatorname{sion}^{2}$ and resolution of the instruments used. A systematic noise floor value of $50 \mathrm{ppm}$ was adopted for the simulations following the framework outlined in Fortney et al. (2013) with additional photon noise estimated using the MRS throughput (Glasse et al. 2010). As discussed in Sect. 3.3, the presence of a strong optical slope in the transmission spectrum of HD 189733b makes it a primary candidate for condensate cloud detection in the wavelength regime covered by JWST. Figure 9 shows three of our cloud condensate models plotted to a resolution of $R \sim 50$ across channels $1-3$ and $R \sim 30$ in channel 4 . The spectra are binned significantly in each of the MIRI channels to increase the photon count at each wavelength therefore reducing the uncertainty of each wavelength bin.

MIRI is ideal to detect the condensate vibrational mode features given in Table 3 where most condensates have distinguishable features which can rise above the expected levels of the gaseous molecular features, with sulphide and chloride condensates a notable exception. Using the example condensates shown

\footnotetext{
2 These estimates can be improved upon with an officially released specific exposure time calculator and increased understanding of the instrument systematics.
}

for the atmosphere of HD 189733b, transmission spectral observations using the two central channels (2 and 3 ) of MIRI/MRS could be vital in distinguishing clouds formed from different condensate species. Each of the $\mathrm{Si}-\mathrm{O}-, \mathrm{Al}-\mathrm{O}-$ and Ti-O-bearing condensate compounds considered have absorption features extending several scale heights above that of the $\mathrm{H}_{2} \mathrm{O}$ dominated molecular model which can be detected and resolved with MIRI. Chanel 2 of the MRS effectively covers the vibrational mode peak of Si-O-bearing compounds with absorption features several scale heights above that of other condensate species shown. Ti-O vibrational mode features are effectively covered by channel 3 of the detector. Al-O-bearing condensate distinction would require observations over multiple channels of MRS, such that species such as $\mathrm{Si}-\mathrm{O}$ and $\mathrm{Ti}-\mathrm{O}$ can be ruled out and the infrared absorption features also matched to the levels observed in the optical.

It is also possible to use the MIRI low resolution spectrometer (LRS) which covers 5-14 $\mu$ m with a $R \sim 100$ (Fortney et al. 2013). LRS encompasses both the major $\mathrm{Si}-\mathrm{O}$ vibrational absorption mode at $\sim 9 \mu \mathrm{m}$ and absorption features generated by photochemical species such as tholins at $\sim 6 \mu \mathrm{m}$. LRS is also advantageous as it requires only a single transit event to cover the entire wavelength range. However, to identify vibrational modes from condensates at wavelengths longer than $\sim 14 \mu \mathrm{m}$, MRS will be required.

\section{Summary}

Clouds are now an increasingly important feature in many hot Jupiter atmospheres. We have investigated the broad spectral properties of clouds, using Mie theory and analytic transmission spectral relations.

We investigate the impact of grain size and distributions on condensate absorption spectra finding that the transmission spectrum can be well represented by the largest particle size in the 
distribution. Additionally, when a strong optical slope is observed in the optical, condensate vibrational mode features become prominent in the infrared associated with clouds composed of small sub-micron sized particles.

Distinguishing cloud composition could in principle help make the distinction between cloud species generated photochemically or through condensation chemistry. We have highlighted spectral features in the infrared generated by the vibrational mode of a condensates major species bond as potential identifiers of cloud compositions in exoplanet atmospheres with the potential for both altitude and wavelength differentiation between species group. While it is difficult with current observations $(<1.7 \mu \mathrm{m})$ to distinguish between different cloud species we find cloud absorption features, caused by the vibrational mode of the major bond species of the condensate, could be present in the infrared, which could help discern different cloud types and constrain particles sizes and altitudes.

The vibrational modes of the various condensates considered in this paper for hot Jupiter atmospheres span a large wavelength regime well into the infrared, with a majority between 9-28 $\mu \mathrm{m}$ where current instruments cannot make transmission measurements.

Of particular interest for this case study is MIRI, JWST's Mid InfraRed Instrument. MIRI has both imaging and spectroscopic capabilities from $5-28.3 \mu \mathrm{m}$ and will have 50 times the sensitivity and seven times the angular resolution of Spitzer, making it a vital instrument for the detection and characterisation of exoplanetary atmospheres as its long wavelength spectral capabilities will be highly sensitive to the cloud properties of transiting exoplanets.

Acknowledgements. H.R.W. acknowledges support from the UK Science \& Technology Facilities Council (STFC). The research leading to these results has received funding from the European Research Council under the European Union's Seventh Framework Programme (FP7/2007-2013)/ERC grant agreement No. 336792. D.K.S. acknowledges support from STFC consolidated grant ST/J0016/1. The authors thank the anonymous referee for their useful feedback and comments. We would also like to thank D.S. Amundsen, B. Drummond, J. Barstow, and K. Heng for useful discussions.

\section{References}

Ackerman, A. S., \& Marley, M. S. 2001, ApJ, 556, 872

Amundsen, D. S., Baraffe, I., Tremblin, P., et al. 2014, A\&A, 564, A59

Anderson, M. R. 2000, Master's Thesis, University of Missouri-Rolla

Andersen, A. C., Mutschke, H., Posch, T., Min, M., \& Tamanai, A. 2006, J. Quant. Spectr. Rad. Transf., 100, 4

Baines, K. H., Hammel, H. B., Rages, K. A., Romani, P. N., \& Samuelson, R. E. 1995, in Neptune and Triton Clouds and hazes in the atmosphere of Neptune, eds. D. P. Cruikshank, M. S.Matthews, \& A. M., Schumann, 489

Batsanov, S., \& Derbeneva, S., 1969, J. Struct. Chem., 10, 510

Begemann, B., Henning, T., Mutschke, H., \& Dorschner, J. 1995, Planet. Space Sci., 43, 1257

Bilger, C., Rimmer, P., \& Helling, C., 2013, MNRAS, 435, 1888

Birkby, J., de Kok, R., Brogi, M., et al. 2013, MNRAS, 436, L35

Bohren, C. F., \& Huffman, D. R. 1983, Absorption and Scattering of Light by Small Particles (New York: Wiley), 82

Burgasser, A. J., Looper, D. L., Kirkpatrick, J. D., Cruz, K. L., \& Swift, B. J. 2008, ApJ, 674, 451

Burrows, A., \& Sharp, C. M., 1999, ApJ, 512, 843

Carlson, B. E., Rossow, W. B., \& Orton, G. S. 1988, J. Atm. Sci., 45, 2066

Cruikshank, D. P., Imanaka, H., \& Ore, C. M. D. 2005, Adv. Space Res., 36, 178

Cushing, M. C., Roellig, T. L., Marley, M. S., et al. 2006, ApJ, 648, 614

Day, K. L. 1981, ApJ, 246, 110

Dorschner, J., Begemann, B., Henning, T., Jaeger, C., \& Mutschke, H. 1995, A\&A, 300, 503

Draine, B. T. 2003, ARA\&A, 41, 241

Ebel, D. S., \& Grossman, L. 2000, Geochim. Cosmochim. Acta, 64, 339

Egan, W. G., \& Hilgeman, T. 1975, AJ, 80, 587
Evans, D., \& Hubbard, W. 1972, Nature, 240, 162

Evans, T. M., Pont, F., Sing, D. K., et al. 2013, ApJ, 772, L16

Fortney, J. J., 2005, MNRAS, 364, 649

Fortney, J. J., Shabram, M., Showman, A. P., et al. 2010, ApJ, 709, 1396

Fortney, J. J., Mordasini, C., Nettelmann, N., et al. 2013, ApJ, 775, 80

Gibson, N. P., Aigrain, S., Barstow, J. K., et al. 2013, MNRAS, 436, 2974

Gillet, P., Guyot, F., Price, G. D., Tournerie, B., \& Le Cleach, A. 1993, Phys. Chem. Minerals, 20, 159

Glasse, A. C. H., Bauwens, E., Bouwman, J., et al. 2010, in The throughput and sensitivity of the JWST mid-infrared instrument, SPIE Conf. Ser., 7731

Glassgold, A., \& Graham, J. 2008, UC Berkeley - ISM: Lecture 06 - 08

Grillmair, C. J., Burrows, A., Charbonneau, D., et al. 2008, Nature, 456, 767

Grossman, L. 1972, Geochim. Cosmochim. Acta, 36, 597

Hansen, J. E., \& Travis, L. D. 1974, Space Sci. Rev., 16, 527

Helling, C. 2007, in Chemical composition of dust clouds in turbulent brown dwarf atmospheres, eds. F. Kupka, I. Roxburgh, \& K. L. Chan, IAU Symp., 239,224

Helling, C. 2008, in Cloud Formation in Giant Planets, Extreme Solar Systems, eds. D. Fischer, F. A., Rasio, S. E. Thorsett, \& A. Wolszczan, 398, 443

Helling, C. 2009a, in Stellar Systems, and the Sun 1094 of Cloud formation in substellar atmospheres, 15th Cambridge Workshop on Cool Stars, ed. E. Stempels, AIP Conf. Ser., 162

Helling, C. 2009b, in Cosmic Dust - Near and Far, The Chemical Evolution of Dust and Gas in Substellar Atmospheres, eds. T. Henning, E. Grün, \& J. Steinacker, ASP Conf. Ser., 414, 125

Heng, K., \& Demory, B.-O. 2013, ApJ, 777, 100

Henning, T., Mutschke, H., \& Jäger, C., 2005, Proc. Int. Astron. Union, 1, 457

Howe, A. R., \& Burrows, A. S. 2012, ApJ, 756, 176

Huffman, D. R., \& Wild, R. L. 1967, Phys. Rev., 156, 989

Huitson, C. M., Sing, D. K., Vidal-Madjar, A., et al. 2012, MNRAS, 422, 2477

Huitson, C., Sing, D. K., Pont, F., et al. 2013, MNRAS, 434, 3252

Kangarloo, H. 2010a, ICMEE2010, 2nd Int. Conf.

Kangarloo, H. 2010b, ICMEE2010, 2nd Int. Conf.

Khare, B., Sagan, C., Arakawa, E., et al. 1984, Icarus, 60, 127

Khare, B., Bakes, E., Cruikshank, D., \& McKay, C. 2001, Adv. Space Res., 27, 299

Knutson, H. A., Charbonneau, D., Allen, L. E., et al. 2007, Nature, 447, 183

Knutson, H. A., Lewis, N., Fortney, J. J., et al. 2012, ApJ, 754, 22

Koike, C., Kaito, C., Yamamoto, T., et al. 1995, Icarus, 114, 203

Kröger, F., \& Meyer, H. 1954, Physica, 20, 1149

Lecavelier Des Etangs, A., Pont, F., Vidal-Madjar, A., \& Sing, D. 2008, A\&A, 481, L83

Lee, J.-M., Irwin, P. G. J., Fletche, L. N., Heng, K., \& Barstow, J. K. 2014, ApJ, 789,14

Lehnert, N., Neese, F., Ho, R. Y., Que, L., Solomon, E. I., 2002, J. Amer. Chem. Soc., 124, 10810

Li, A., \& Draine, B. T. 2001, ApJ, 550, L213

Li, A., \& Draine, B. T. 2002, ApJ, 564, 803

Liang, M.-C., Seager, S., Parkinson, C. D., Lee, A. Y.-T., \& Yung, Y. L. 2004, ApJ, 605, L61

Liou, K.-N. 2002, An introduction to atmospheric radiation (Academic press), 84

Lodders, K. 1999, ApJ, 519, 793

Lodders, K. 2003, ApJ, 591, 1220

Lodders, K. 2010, Exoplanet Chemistry (Wiley), 157

Lodders, K., \& Fegley Jr B. 2006, in Astrophysics Update 2 (Springer), 1

M. Meinecke, B. P. 2005, Database of Optical Constants for Cosmic Dust

Marley, M. S., Fortney, J., Seager, S., \& Barman, T. 2007, Protostars and Planets V, 733

Marley, M. S., Ackerman, A. S., Cuzzi, J. N., \& Kitzmann, D. 2013, Clouds and Hazes in Exoplanet Atmospheres, 367

Martonchik, J. V., \& Orton, G. S., 1994, Appl. Opt., 33, 8306

McCullough, P. R., Crouzet, N., Deming, D., \& Madhusudhan, N. 2014, ApJ, 791,55

Morley, C. V., Fortney, J. J., Marley, M. S., et al. 2012, ApJ, 756, 172

Morley, C. V., Fortney, J. J., Kempton, E. M.-R., et al. 2013, ApJ, 775, 33

Morley, C. V., Marley, M. S., Fortney, J. J., et al. 2014, ApJ, 787, 78

Moses, J., Madhusudhan, N., Visscher, C., \& Freedman, R., 2013, ApJ, 763, 25

Nascimbeni, V., Piotto, G., Pagano, I., et al. 2013, A\&A, 559, A32

Nixon, C., Achterberg, R., Romani, P., et al. 2010, Planet. Space Sci., 58, 1667

Palik, E. D. 1998, Handbook of Optical Constants of Solids: Index. Access Online via Elsevier, 3

Parmentier, V., Showman, A. P., \& Lian, Y. 2013, A\&A, 558, A91

Pont, F., Knutson, H., Gilliland, R. L., Moutou, C., \& Charbonneau, D. 2008, MNRAS, 385, 109

Pont, F., Sing, D., Gibson, N., et al. 2013, MNRAS, 432, 2917

Posch, T., Kerschbaum, F., Fabian, D., et al. 2003, ApJS, 149, 437 
H. R. Wakeford and D. K. Sing: Transmission spectral properties of clouds for hot Jupiter exoplanets

Querry, M. 1987, Technical report, Optical constants of minerals and other materials from the millimetre to the ultraviolet, Chemical Research, Development and Engineering Center, US Army Armament Munitions Chemical Command

Ramirez, S., Coll, P., da Silva, A., et al. 2002, Icarus, 156, 515

Redfield, S., Endl, M., Cochran, W. D., \& Koesterke, L. 2008, ApJ, 673, L87

Rice, S. A., \& Klemperer, W. 2004, J. Chem. Phys., 27, 573

Rothman, L. S., Gordon, I. E., et al. 2009, J. Quant. Spectrosc. Radiat. Transf., 110,533

Saniger, J. 1995, Mat. Lett., 22, 109

Seager, S. 2010, Exoplanet atmospheres: physical processes (Princeton University Press)

Sharp, C. M., \& Burrows, A. 2007, ApJS, 168, 140

Showman, A. P., Cooper, C. S., Fortney, J. J., \& Marley, M. S., 2008, ApJ, 682, 559
Sing, D. K., Désert, J.-M., Lecavelier Des Etangs, A., et al. 2009, A\&A, 505, 891

Sing, D. K., Pont, F., Aigrain, S., et al. 2011, MNRAS, 416, 1443

Sing, D. K., Lecavelier des Etangs, A., Fortney, J. J., et al. 2013, MNRAS, 436, 2956

Sing, D. K., Wakeford, H. R., Showman, A. P., et al. 2014, MNRAS, submitted Southworth, J. 2010, MNRAS, 408, 1689

Sudarsky, D., Burrows, A., \& Hubeny, I. 2003, ApJ, 588, 1121

Torres, G., Winn, J. N., \& Holman, M. J. 2008, ApJ, 677, 1324

Wakeford, H., Sing, D., Deming, D., et al. 2013, MNRAS, 435, 3481

Yurchenko, S. N., \& Tennyson, J. 2014, MNRAS, 440, 1649

Zahnle, K., Marley, M. S., Freedman, R. S., Lodders, K., \& Fortney, J. J. 2009, ApJ, 701, L20

Zeidler, S., Posch, T., Mutschke, H., Richter, H., \& Wehrhan, O. 2011, A\&A, 526, A68 